\title{
La geografía escolar y las ciencias sociales: el caso de la secundaria oficial en España'
}

\author{
School Geography and the Social Sciences: the Case of \\ Spain's Public Secondary Schools
}

Israel Cabeza Morales²

\author{
Resumen
}

El presente trabajo, producto de una corta estancia de investigación en el CCHS-CSIC, constituye un análisis sobre la manera en que son entendidas las ciencias sociales en la educación secundaria obligatoria en Madrid, deteniéndose particularmente en el papel desempeñado por la Geografía. En él se recogen las percepciones de profesores y distintos profesionales de áreas relacionadas con las ciencias sociales, revisiones de la normativa, críticas, pero, ante todo, se plantean algunos interrogantes, cuyo objeto es causar eco en quienes se consideran vinculados no solo a este cuerpo disciplinar, sino a la educación en general.

\section{Palabras clave}

Ciencias sociales, Geografía, educación secundaria, didáctica, contenidos.

\section{Abstract}

This paper, which is the result of a short research internship in the CCHS-CSIC, is an analysis on how social sciences in Madrid's Secondary Education are understood. Particular attention is paid to the role played by geography. The paper takes into consideration the testimonies of teachers and other professionals from the field of social sciences, documents and statements related to curricular standards, and some critical reviews. Above all, some questions are posited, whose purpose is to impact in those who consider themselves part not only of this field of study, but of education in general.

Key words

Social sciences, geography, high school, teaching, curriculum.

Artículo recibido el 25 Abril de 2011 y aprobado el 30 Marzo de 2012

1 Informe producto de una estancia de investigación realizada durante el verano de 2009, en el Instituto de Lengua, Literatura y Antropología ILLA, del Centro de Ciencias Humanas y Sociales CCHS, adscrito al Consejo Superior de Investigaciones Científicas CSIC , en Madrid (España).

2 Magister en Geografía del Instituto Geográfico Agustín Codazzi. Profesor de la Universidad Pedagógica y Tecnológica de Colombia, israel.cabeza@uptc.edu.co. 
Saber pensar el espacio. Y saber pensar el espacio, tener un razonamiento geográfico, no para soñar con las estrellas, es pensar el espacio con una visión política de las cosas, saber pensar para actuar más eficazmente y para comprender mejor lo que puede pasar.

Yves Lacoste

\section{Introducción}

Este trabajo trata de las ciencias sociales como gran área del saber escolar. Su análisis se hará desde los niveles de la educación secundaria obligatoria (ESO), por considerar tal etapa de gran relevancia para la construcción de una conciencia social en los educandos, determinante en su proceso formativo como sujetos sociales. Se ha elegido trabajar particularmente con la Geografía, específicamente su relación con las ciencias sociales en la ESO oficial de Madrid, a partir de la recolección y el tratamiento de información, realizado en algunos centros educativos de la ciudad.

Al inicio del ejercicio investigativo, el objetivo era estudiar el estado de la Geografía escolar en relación con las ciencias sociales, en la educación secundaria oficial en Madrid, a través del análisis de sus contenidos y las percepciones que se tienen de esta desde el aula. A este objetivo nos hemos aproximado en particular desde la interpretación de la articulación que pudiera existir entre los contenidos de Geografía escolar establecidos por el Ministerio de Educación español, y que son parte del campo general de las ciencias sociales, y los que son efectivamente impartidos en las aulas, dando prioridad al siguiente interrogante: ¿cuál es la relación entre la Geografía escolar y las ciencias sociales en la ESO de Madrid?

La planeación inicial se vio afectada por limitaciones de tiempo y de acceso a las instituciones educativas para la época del año en que se realizó la estancia académica (transición verano-otoño), pero se logró un nivel de trabajo que permitió la construcción de múltiples reflexiones y el surgimiento de nuevos cuestionamientos sobre la pregunta problema. Cabe mencionar que para el desarrollo de este trabajo se contó con la colaboración del
CCHS del CSIC 1 y el apoyo de algunos docentes del Departamento de Didáctica de las Ciencias Sociales en la Facultad de Educación de la Universidad Complutense de Madrid², quienes aportaron con orientaciones y comentarios para el mejor desarrollo del proyecto.

En el marco de las ciencias sociales hay mucho por discutir. Dadas las presiones que imponen las exigencias de productividad y los enfoques positivistas de construcción de conocimiento, estas disciplinas se han visto obligadas a redefinirse y a clarificar su pertinencia como ciencias. El desarrollo de este cuerpo disciplinar ha llevado a la consolidación de diversas posturas: por un lado, algunos aseguran la necesidad de romper barreras e interconectar disciplinas para generar saberes complejos ajustados a las exigencias de la sociedad; por otro lado, hay quienes ven en esto riegos disciplinares, híbridos conceptuales, que pueden ser perjudiciales para las mismas, para su cientificidad, un aspecto bastante relativo (Pérez et al, 2002).

Quizás por esto, desde la segunda mitad del siglo pasado se ha pensado en la necesidad de clarificar el concepto de ciencias sociales. Esta tarea no es nada fácil, por lo que algunos autores 3 han sugerido que debería hablarse de "estudios sociales" en vez de "ciencias sociales", al menos a nivel escolar. Lo anterior explica la encrucijada de esta disciplina y con ella del concepto de lo humano que han albergado; ese sentir social que hace mención a la compresión de realidades. Ante lo anterior, surgen interrogantes como ¿será que tal denominación merece ser aclarada? ¿Cómo haríamos con los recientes aportes que se dan hacia lo social, desde diversas disciplinas? ¿Es verdaderamente pertinente romper fronteras? ¿No necesita tal ruptura una demarcación anti-caótica?

1 Por parte del Centro de Ciencias Humanas y Sociales (CCHS), del Consejo Superior de Investigaciones Científicas CSIC, se resalta la tutoría de la doctora Margarita del Olmo Pintado y la asesoría de la doctora Matilde Fernández Montes.

2 Especialmente la colaboración de la profesora Caridad Hernández y el profesor Miguel Ángel Sandoya.

3 Ver las apreciaciones sobre las ciencias sociales en Immanuel Wallerstein y por John Jarolimek, son discursos aparentemente distintos, pero ligados a una des-estigmatización de la noción de ciencias sociales. 
¿Cuántas son las disciplinas que conciernen al marco de las ciencias sociales? ¿Es necesaria la diferenciación entre ciencias sociales y humanas? ¿Acaso lo humano no es social y/o viceversa? Por estas y otras razones, las ciencias sociales siguen siendo un tema vigente.

Sobre cuestionamientos como los anteriores se sustenta el presente trabajo, que no pretende redefinir el concepto de ciencias sociales a nivel académico, sino hacer una aproximación significativa a la manera como estas son entendidas en la escuela, deteniéndose particularmente en la Geografía, una disciplina cuya relación con las ciencias sociales en general es asumida de manera distinta en los sistemas escolares de cada país. Para el caso de España, podríamos asegurar que por la forma como se denomina en la normatividad educativa ${ }^{4}$, en la asignatura -"Ciencias Sociales, Geografía e Historia”-, la Geografía es considerada algo externo del área de trabajo. No obstante tal denominación, esta se constituye como eje o base, al igual que la historia, para dar una idea del concepto de ciencias sociales. Lo anterior explicaría por qué para muchos jóvenes estudiantes, e incluso universitarios, esta denominación es equivalente a Geografía e Historia.

Si bien se ha dicho que estas dos disciplinas son claves para entender lo social y que como ellas ninguna otra ha de explicarlo, esto no justifica la potestad de opacar o aislar a las otras del conocimiento escolar como disciplinas válidas para la comprensión de las ciencias sociales. Con esta consideración no se propone una ampliación de contenidos, sino una reorientación tanto en la formación del profesorado como en la estructuración de ciertos contenidos donde la transversalidad esté clara y no dé la apariencia de una simple adición disciplinar, lo que permitiría a los estudiantes ser conscientes de la existencia de esas otras disciplinas.

Aquí resulta interesante el caso de la Geografía escolar española, que ha sido trabajada por geógrafos y educadores 5 , quienes, preocupados por el

\footnotetext{
4 Tanto para el país en general como para la normativa especial de la Comunidad de Madrid.

5 Podría hablarse de reconocidos profesores como Xosé Manuel
}

avance de la disciplina en este país e interesados por la mejora de las condiciones de su enseñanza, se han dedicado a investigar y a reflexionar sobre su desarrollo a nivel escolar. Valdría la pena resaltar que tales trabajos en su mayoría establecen relaciones con la historia, en el sentido en que explican la manera como se manejaba la enseñanza de la disciplina en determinado tiempo, en la educación primaria, secundaria y de bachillerato, cuando la Geografía era una disciplina escolar impartida de manera independiente de las ciencias sociales y de la Historia, diferente a la forma como se imparte según la denominación actual de la asignatura.

Cabe mencionar que en España esta disciplina estuvo muy cuestionada entre los años 50 y los $80^{6}$, dado su relativo atraso en relación con otros países del mundo, proyectado en las escuelas y en las universidades, atraso que, según algunos académicos (Luis, 1985), todavía es evidente en la disciplina. Quizás una explicación sea la marcada diferenciación de enfoques o corrientes en la que se ubicaban los geógrafos españoles, mayoritariamente de tipo humano, poco cuantitativo, lo que de una u otra forma incide en la formación universitaria y por tanto en la escuela; lo particular es que, siendo así el desarrollo de la Geografía, debería tender hacia su consolidación como ciencia social, tal como ha sido definida con el paso del tiempo, gracias a las reflexiones epistemológicas y el revisionismo metodológico.

Varias son las revisiones que se han hecho a los objetivos, contenidos y metodologías para el desarrollo de los procesos de enseñanza-aprendizaje de la Geografía. Entre ellas podríamos hablar desde las valoraciones epistémicas y/o de corrientes de pensamiento, incluyendo la importancia de elementos como la causalidad, si bien: "la Geografía es el estudio de los espacios de la tierra según sus diferencias causales"7 . Lo anterior deja ver la marcada

Souto, Alberto Luís Gómez, Pedro Plans Sanz, Horacio Capel, Pilar Benejam, entre muchos otros (Ver bibliografía).

6 Ver: Luis Gómez, A. (1985). La Geografía en el Bachillerato Español (1836-1970). Barcelona: Universitat de Barcelona.

7 Alfred Hettner, citado por Bailey, P. (1981). Didáctica de la Geografía. Madrid: Editorial Cincel. 
influencia del saber pedagógico en la enseñanza de la Geografía, en textos de didáctica de esta disciplina específica (publicaciones poco recientes), así como en textos sobre didáctica de las ciencias sociales, en los que se incluye a la Geografía, los cuales, son mayoritarios y resultan más vigentes.

Adicionalmente, la pertinencia de esta disciplina para la formación de los jóvenes, la estructura procedimental y temática, las herramientas y la importancia de su uso, han sido aspectos estudiados y sus resultados han resaltado la importancia de "saber Geografía para enseñar Geografía".

Por otro lado está la necesidad de "saber enseñar" pero, ante todo, de tener una visión de mundo que no se cierre a nuevas ideas, que resalte la posibilidad potencial de la Geografía, que comprenda su labor y permita, a través de su enseñanza, que los jóvenes analicen lo que está más allá de la escuela y los haga sentir hijos del planeta, ciudadanos de este mundo, para lo cual las aptitudes rígidas se convierten en un inconveniente.

\section{La ESO y las ciencias sociales en el sistema educativo español}

El sistema educativo español, o mejor, su estructura, se ha reajustado recientemente de acuerdo con las directrices de la Unión Europea. Podríamos decir que ha reestructurado su organización para estar al nivel de lo que implica la articulación con el resto de la Unión, la Ley Orgánica de Educación, cuya fecha de publicación es del 3 de mayo de 2006, plantea la directriz general para el manejo de la educación española, que hace alusión a la educación secundaria obligatoria (ESO), considerando que:

2. La finalidad de la educación secundaria obligatoria consiste en lograr que los alumnos y las alumnas adquieran los elementos básicos de la cultura, especialmente en sus aspectos humanístico, artístico, científico y tecnológico; desarrollar y consolidar en ellos hábitos de estudio y de trabajo; prepararles para su incorporación a estudios posteriores y para su inserción laboral y formarles para el ejercicio de sus derechos y obligaciones en la vida como ciudadanos ${ }^{8}$.

8 Ley Orgánica 2/2006 de Educación, en el Titulo I, Capítulo III, articulo 22, literal 2
Lo anterior es el reflejo más claro de la intencionalidad e importancia de esta etapa educativa, cuya duración es de cuatro años y que al finalizar brinda la posibilidad de ingreso al bachillerato. Esta última es una etapa de dos años en la que el estudiante se forma para la competencia técnica, artística y/o laboral, así como para la inserción en la educación superior. En la parte del artículo ya citado se muestra una intencionalidad educativa ${ }^{9}$ diferente a la proyectada por la estructura general de las múltiples enseñanzas que establece el gobierno español.

Se evidencia una articulación con el ideal de hombre y ciudadano, una noción de formación para la vida en sociedad que podría llevar a pensar que en la ESO se forja la construcción del individuo como sujeto social, entendiendo tal denominación como una propensión ideal de la humanidad hacia un ser potencialmente humanizador, con una enseñanza no desligada de la importancia de los contenidos conceptuales $^{10}$. Esto es de gran significado para el fenómeno del trabajo por competencias ${ }^{11}$, un objetivo transversal para toda la Unión Europea.

Un factor trascendental es el hecho de que en la orden 3320-01/2007 del 20 de junio de 2007, expedida por el Consejero de Educación, mediante la cual se regulan la implantación y la organización de la Educación Secundaria Obligatoria derivada de la Ley Orgánica 2/2006 de Educación para la Comunidad de Madrid, se deja claridad sobre el grado de compromiso al que deben apuntar los actores educativos en su conjunto, al establecer que "las tecnologías de la información y la comunicación estarán integradas en el currículo"12.

Tal enunciado reitera una intención de hacer uso de los recursos tecnológicos en cada una de las asignaturas, aunque exista una de "tecnologías". Esta

9 Con este término pretendo hacer alusión al carácter procedimental y/o institucional, poco significativo, desde el que se perciben los distintos niveles de educación, muchas veces aislados de un ideal verdaderamente formativo al que debe vincularse lo educativo.

10 Algo que plantea un cambio de posición, dado que la normativa anterior (LOGSE), a diferencia de la actual, cambiaba su prioridad de lo conceptual a la primacía de lo procedimental y actitudinal, de lo que aún quedan muchos rastros.

11 Ver: Ley de Calidad de la Educación de 2002.

12 Órden 3320-01/2007, de 20 de junio, Artículo 3, apartado 5 
inclusión del componente tecnológico, más que una integración o supresión de conocimientos básicos, es el reflejo del compromiso con la educación española, lo que implica una preocupación por la cualificación constante del profesorado y a la vez un interés por el reconocimiento de su labor ${ }^{13}$.

Por otro lado, en la Ley Orgánica de Educación también se hace referencia a dos aspectos claves: la autonomía que tienen las comunidades autónomas para regular procesos como el establecimiento de directrices curriculares ${ }^{14}$ y el marco de la interculturalidad y la igualdad de derechos como fines de la educación, para responder a un ideal nacional y resaltar el interés por la colaboración territorial y la concertación para la planificación de tal proceso.

Es tal la claridad de la Ley Orgánica, que establece las materias que deben cursar los jóvenes de la ESO en cada uno de los cuatro niveles, en los que las ciencias sociales tienen un espacio, pero la denominación como asignatura se hace a través de la expresión "Ciencias Sociales, Geografía e Historia". Esta materia, a juzgar por su nombre, podría develar un delineamiento interesante, pues da la impresión de que el área se trabaja como un constructo, que es complementado por la Geografía y la Historia. En ese caso sería pertinente preguntarse: ¿Estas dos disciplinas son sociales o humanas? O, por lo menos, ¿ ¿a cuál de esos selectos grupos está asignada la Geografía? Lo más adecuado para hacer un acercamiento significativo a esta cuestión, es mirar la manera como se establece el currículo de dicha asignatura, en la Comunidad Autónoma de Madrid, que es el referente base de acuerdo con el tema de este trabajo.

\section{Asignaturas con vinculación a las ciencias sociales}

Vale la pena aclarar que si comprendemos esa denominación de ciencias sociales como algo a lo que adicionalmente están vinculadas la Geografía y la

\footnotetext{
13 Ver: Ley Orgánica 2/2006 de Educación, Título III, capítulos III y IV (publicada en el BOE, 4 de mayo de 2006)

14 Ver: Ley Orgánica 2/2006 de Educación, Título V, capítulos I y V (publicada en el BOE, 4 de mayo de 2006)
}

Historia, sin ser tal vinculación razón para que se entiendan las mismas como un todo, es necesario reiterar que la formación en derechos humanos se establece como una asignatura más, que deberán cursar los jóvenes una sola vez, entre el primer y tercer curso de la ESO, establecido así:

3. En uno de los tres primeros cursos todos los alumnos cursarán la materia de Educación para la Ciudadanía y los Derechos Humanos en la que se prestará especial atención a la igualdad entre hombres y mujeres ${ }^{15}$.

¿Acaso eso no es producto de las ciencias sociales? Valdría la pena cuestionar la relación de esta materia con las ciencias sociales, sobre todo en la medida en que dicha norma en otro de sus apartados hace alusión a la obligación de que en la ESO se curse una asignatura más, definida como Educación ÉticoCívica16, cuyo enfoque principal será la igualdad de género. Esto hace preguntarse sobre el límite hasta donde llega la idea de esta área del conocimiento en España. ¿Será que tal fragmentación obedece a una visión rigurosa del cuerpo de académicos que se dedican tanto a las ciencias sociales como humanas, o propia del profesorado de la ESO? ¿Por qué se habla de ciencias sociales, si en España la historia no es una ciencia social sino humana, a nivel científico?

Si la historia está ligada a las ciencias sociales, por lo menos en su denominación como asignatura escolar, entonces cabe decir que, afortunadamente para los historiadores y amantes de la historia, esta tiene presencia externa a la asignatura de "Ciencias Sociales, Geografía e Historia”. Prueba de ello es la manera como se sustenta la enseñanza religiosa en la ESO, reconocida como necesaria en la ley orgánica en la que es definida como asignatura de impartición obligatoria por parte de las instituciones, y de carácter voluntario para los alumnos ${ }^{17}$.

15 Ley Orgánica 2/2006 de Educación, Titulo 1, capitulo 3, artículo 24, literal 3. (publicada en el BOE, 4 de mayo de 2006)

16 Ley Orgánica 2/2006 de Educación, Titulo 1, capitulo 3, articulo 25, literal 4. (publicada en el BOE, 4 de mayo de 2006)

17 Aunque la tradición de España sea católica, resulta particularmente curiosa la manera como se hace referencia a la enseñanza religiosa. (Ver: Ley Orgánica 2/2006 de Educación, Disposición adicional segunda). 
La verdadera particularidad y posible razón para establecer el vínculo de la religión con las ciencias sociales no radica solo en el hecho de que esta área se encuentre ubicada en la mayoría de las facultades de educación, dentro de los departamentos de ciencias sociales ${ }^{18}$, sino en que, al revisar la orden por la que la Comunidad de Madrid regula la implantación de la Ley Orgánica de Educación, que hace una articulación con lo histórico, al definirla como "Religión Católica y de Historia y Cultura de las Religiones ${ }^{19}$ ”, se podría mencionar que esa misma orden establece a las asignaturas mencionadas una intensidad horaria particular que debería motivarnos a reflexionar sobre la idea de lo que entendemos por ciencias sociales. Veamos:

\section{Asignaturas relacionadas con las ciencias sociales}

Tabla 1: Asignación horaria de asignaturas relacionadas con las CS en la $\mathrm{ESO}^{20}$

\begin{tabular}{|l|l|l|l|l|}
\hline \multirow{2}{*}{ Asignatura } & \multicolumn{4}{|l|}{ Periodo lectivo } \\
\cline { 2 - 6 } & $1^{\circ}$ & $2^{\circ}$ & $3^{\circ}$ & $4^{\circ}$ \\
\hline Ciencias Sociales, Geografía e Historia & 3 & 4 & 3 & 3 \\
\hline $\begin{array}{l}\text { Religión/Historia y cultura de las Reli- } \\
\text { giones }\end{array}$ & 1 & 2 & 1 & \\
\hline $\begin{array}{l}\text { Educación para la ciudadanía y los de- } \\
\text { rechos humanos }\end{array}$ & & 1 & & \\
\hline Educación ético-cívica & & & & 1 \\
\hline
\end{tabular}

El cuadro anterior constituye una fuente válida para entender cómo se perciben las ciencias sociales en la educación española, particularmente en la ESO, que es el punto medio entre la vida laboral, académica o profesional, ya que desde allí se forjan los caminos y directrices en los que se enmarcan los jóvenes hacia el desarrollo del bachillerato. Esto la constituye como una etapa crucial para el

18 Quizás debido a que los maestros de educación primaria ven en su formación algo de educación religiosa, dado que deben impartirla.

19 Ver: ORDEN 3320-01/2007, artículo 8.

20 Elaborada con base en el anexo de la ORDEN 3320-01/2007, de 20 de junio, del Consejero de Educación, por la que se regulan para la Comunidad de Madrid la implantación y la organización de la educación secundaria obligatoria derivada de la Ley Orgánica 2/2006, de 3 de mayo, de Educación. periodo formativo de quienes desde ahí construyen su visión de sí mismos como hombres y mujeres, como ciudadanos. Pasemos ahora a un análisis de los contenidos, aspecto más significativo que el de las denominaciones.

\section{Una mirada a los contenidos de Ciencias Sociales, Geografía e Historia}

Tantas son las formas y modos de ver y evaluar los contenidos de una asignatura, que lo primordial es mirar la norma, como medida inicial, que es lo que se ha venido haciendo en el presente trabajo. Para continuar, nuestro referente clave es el Decreto 23/2007, por el cual el Consejo de Gobierno de la Comunidad de Madrid establece el currículo de la ESO. Esto es significativamente beneficioso, pues clarifica hacia dónde se deben direccionar las velas de esa tripulación que emprende una travesía, curso tras curso. Algunos pueden percibirlo como algo demasiado condicionante, pero puede entenderse como muy claro y necesario para el desarrollo de los procesos de enseñanza-aprendizaje a cualquier nivel.

A pesar de las aparentes bondades del establecimiento del currículo, tal como lo hace la norma, hay que valorar, sopesar y re-evaluar el modo como se plasman las temáticas, los bloques, su articulación y secuencialidad. Algunas cosas no son fáciles; quizás por eso la educación es considerada por muchos una tarea tediosa, que pasa de la reestructuración constante a la crítica mordaz, a la que se ven condenados quienes felizmente se embarcan en esas aguas, con la idea de gestar o impulsar cambios. Pero antes hay que comprender la complejidad de la profesión docente; de ahí la responsabilidad de los estudiosos de las ciencias sociales que han de repensarse un mundo, que la realidad les impide muchas veces recrear, lo que constituye la base para entender los ires y venires en la comprensión de lo social, para seguir siendo maestros, académicos y/o ciudadanos.

Para el establecimiento de los contenidos de cada asignatura, en el decreto antes mencionado se plantea una introducción, la contribución de determinada materia a la adquisición de competencias básicas y los objetivos, que afectan tanto a historia 
como Geografía y los comunes. Resulta curioso que en la introducción, para el caso de las Ciencias Sociales, Geografía e Historia, no se haga referencia al área general como tal. Sin embargo, sí se menciona la importancia de la Geografía y la Historia para los países, las sociedades y la humanidad en general, lo que lleva a entender algo que quizás algunos consideran errado: Geografía e Historia forman parte de las ciencias sociales.

En la introducción se explica además la potencialidad de estas disciplinas como base para entender el pasado, presente y futuro de la humanidad. Explica qué contenidos de Geografía e Historia serán vistos curso a curso, desde un panorama general, a través de temas centrales para cada disciplina. Sin embargo, la directriz temática parece apuntar a otro rumbo, particularmente en el tercer y cuarto curso, donde la asignatura sigue teniendo el mismo nombre. Veamos:

\section{Temas generales de Geografía e Historia en la ESO}

Tabla 2: Tópicos generales de la ES0 en ciencias sociales 21

\begin{tabular}{|l|l|l|}
\hline Curso & \multicolumn{1}{|c|}{ Geografía } & \multicolumn{1}{c|}{ Historia } \\
\hline $1^{\circ}$ & $\begin{array}{l}\text { Estudio de la Tierra y los } \\
\text { medios naturales, con es- } \\
\text { pecial atención a la rela- } \\
\text { ción entre el hombre y el } \\
\text { espacio físico. }\end{array}$ & $\begin{array}{l}\text { Estudio de las socieda- } \\
\text { des prehistóricas, prime- } \\
\text { ras civilizaciones y Edad } \\
\text { Antigua, con la finalidad } \\
\text { de que el alumno conoz- } \\
\text { ca la configuración de las } \\
\text { primeras civilizaciones. }\end{array}$ \\
\hline $2^{\circ}$ & $\begin{array}{l}\text { Estudio de las caracterís- } \\
\text { ticas y tendencias de la } \\
\text { población, en especial la } \\
\text { española y la europea, su } \\
\text { estructura y diversidad. }\end{array}$ & $\begin{array}{l}\text { Estudia la Edad Media y y } \\
\text { la Edad Moderna, man- } \\
\text { teniendo el orden crono- } \\
\text { lógico. }\end{array}$ \\
$\begin{array}{l}\text { Predominan los aspectos geopolíticos y económicos. } \\
\text { La mayor parte de los contenidos se abordan desde } \\
\text { el análisis de la organización política y el espacio geo- } \\
\text { gráfico de los grandes ámbitos del mundo, de la Unión } \\
\text { Europea y de España, así como las transformaciones } \\
\text { y problemas de un mundo interdependiente y de la cre- } \\
\text { ciente globalización, con especial referencia a las des- } \\
\text { igualdades en el desarrollo humano. Se concluye con } \\
\text { el análisis del espacio geográfico europeo y español, } \\
\text { con especial referencia a la diversidad geográfica de } \\
\text { España y de la Comunidad de Madrid. }\end{array}$ \\
\hline
\end{tabular}

21 Elaborada con base en el Decreto 23/2007, del 10 de mayo, del Consejo de Gobierno, por el que se establece para la Comunidad de Madrid el currículo de la Educación Secundaria Obligatoria.

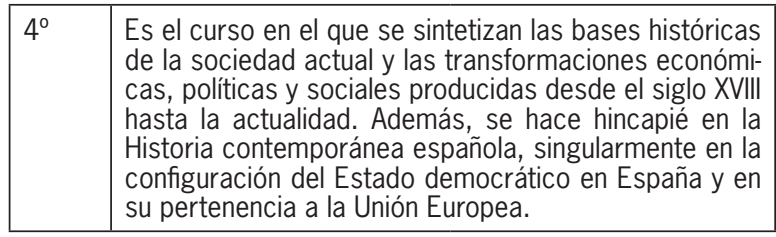

La tabla nos permite ver la manera como están estructuradas esas dos disciplinas, Geografía e Historia, como el eje transversal de lo que son las ciencias sociales, desde la explicación textual de la comprensión de las sociedades, su evolución, el análisis integral de la realidad, comprendiendo la funcionalidad tiempo-espacio, que se hace tanto en la introducción como en la contribución de la asignatura a la adquisición de competencias básicas. En ella, incluso se habla de la potestad de las ciencias sociales para la articulación con otros saberes y se pone en un nivel muy alto la transversalidad de las mismas, aunque a su vez se muestre que hay un juego de distribución disciplinar más o menos homogéneo, en el que ni la Historia ni la Geografía salen perdiendo. Así, aparentemente, quedan catalogadas como ciencias sociales, pese a que otros aseguren que no lo son. De todas formas, en el texto del decreto, la idea de ciencias sociales transmitida no se alcanza a desarrollar en plenitud con los contenidos de la tabla anterior.

Algo positivo en la forma como está organizada la norma es que se emplea la estructuración curricular de acuerdo con los bloques de contenidos, los cuales son como una directriz por la que el profesorado se guía en el momento de establecer su temario. En cada curso, antes de ver los bloques de contenidos de Geografía e Historia, se establecen bloques comunes; es allí donde se aprecia la magia, la articulación disciplinar y el universo de posibilidades formativas que se generan desde y hacia estas áreas del saber.

Así como hay contenidos y objetivos comunes, también los hay disciplinares. Estos están planteados de modo particular: los comunes antes que los disciplinares, lo cual es realmente significativo en la medida en que proyecta una noción ideal de ciencias sociales, un verdadero ejercicio interdisciplinar, al menos en la norma. A pesar de esto, a nivel académico, desde los centros de investigación, se dice que 
la Geografía y la Historia no pertenecen a este grupo sino al de las ciencias humanas, lo que se ratifica en el nombre de la asignatura en la ESO.

Resulta grave tratar de entender la situación de la Geografía, que tiene como una de sus dificultades el hecho de tener en el tercer curso de la ESO una estructura de contenidos en la que cuatro de los cinco bloques temáticos le son concernientes. Este curso es el que tiene contenidos más extensos en toda la ESO y con una asignación de tiempo muy similar a la de otros. Esto también tiene la "ventajosa desventaja", si se puede definir como tal, de tener un bloque temático en el primer curso con contenidos muy similares a los de Biología y Geología o ciencias naturales ${ }^{22}$, asignaturas en las que aspectos como la tierra y el universo, la atmósfera o características de la litosfera están presentes.

Vale decir que esto puede convertirse en ventaja para los alumnos y el profesorado, pero solo si los primeros tienen la aptitud ideal y el profesorado aprovecha tal oportunidad para mostrar la temática desde el enfoque eminentemente geográfico, haciendo uso de esta coincidencia para mostrar la interdisciplinariedad y el potencial de la Geografía en particular para trabajar con otras disciplinas. De lo contrario, esto puede ser una amenaza.

\section{Los contenidos de Geografía}

Resulta de gran interés observar la presencia de la Geografía en el área de las ciencias sociales en la ESO. La Tabla 3 nos muestra una distribución armónica y bastante ordenada de los contenidos geográficos que son base para llegar a contenidos propios de las ciencias sociales en cada uno de los niveles o cursos de la ESO. Como ya se mencionó, se encuentran unos bloques comunes, al inicio de los bloques disciplinares de Geografía e Historia. Si observamos más detenidamente, estos bloques

22 Es posible referirse a cualquiera de las dos, ya que las ciencias naturales, en el tercer curso de la ESO, son divididas en dos asignaturas (Biología y Geología, así como Física y Química). Ver: Decreto 23/2007 del 10 de mayo de 2007, expedido por el Consejo de Gobierno, mediante el cual se establece para la Comunidad de Madrid el currículo de la Educación Secundaria Obligatoria (Art. 6, apartado 4) resultarían más significativos si se plantean al final de cada curso, ya que propiciarían una mayor articulación de los temas vistos desde cada disciplina. A pesar de esto, tales bloques comunes no están clarificados temáticamente, sino que aparecen en la norma como acciones o habilidades que el estudiante debe alcanzar y se confunden con los objetivos ${ }^{23}$.

\section{Geografía en los cursos de la $\mathrm{ESO}^{24}$}

Tabla 3: Bloques de Geografía en la ESO

\begin{tabular}{|c|c|c|}
\hline $1^{\circ}$ & $2^{\circ}$ & $3^{\circ}$ \\
\hline \multirow[t]{2}{*}{$\begin{array}{l}\text { Bloque 2. Geografía. } \\
\text { La Tierra y los me- } \\
\text { dios naturales. } \\
\text { 2.1. El planeta Tierra. } \\
\text { 2.2. Los elementos } \\
\text { del medio físico. } \\
\text { 2.4. Los medios na- } \\
\text { turales de Europa. } \\
\text { 2.5. Los medios na- } \\
\text { turales de España. }\end{array}$} & $\begin{array}{l}\text { Bloque 2. Geografía. } \\
\text { La sociedad y sus re- } \\
\text { laciones con la natu- } \\
\text { raleza. } \\
\text { 2.1La población mun- } \\
\text { dial. } \\
\text { 2.2. La explotación de } \\
\text { los recursos naturales. } \\
\text { 2.3. Sociedad y Esta- } \\
\text { do. } \\
\text { 2.4. El espacio urbano. } \\
\text { 2.5. España: población } \\
\text { y recursos naturales. } \\
\text { El Estado. }\end{array}$ & $\begin{array}{l}\text { Bloque 2. Geografía. } \\
\text { Actividades económi- } \\
\text { cas y espacios geo- } \\
\text { gráficos. } \\
\text { 2.1. La actividad eco- } \\
\text { nómica. } \\
\text { 2.2. Actividades y es- } \\
\text { pacios del sector pri- } \\
\text { mario. } \\
\text { 2.3. Actividades y es- } \\
\text { pacios industriales. } \\
\text { 2.4. Las actividades } \\
\text { de los servicios y sus } \\
\text { espacios. } \\
\text { 2.5. Un mundo de ciu- } \\
\text { dades. } \\
\text { 2.6.Actividades y es- } \\
\text { pacios geográficos en } \\
\text { España. } \\
\text { Bloque 3. Geografía. } \\
\text { El mundo actual. } \\
\text { 3.1. El espacio mun- } \\
\text { do. } \\
\text { 3.2. El mundo desarro- } \\
\text { llado. } \\
\text { 3.3. El mundo subde- } \\
\text { sarrollado. } \\
\text { 3.4.Transformaciones } \\
\text { y desequilibrios en el } \\
\text { mundo actual. } \\
\text { Bloque } 4 \text {. Geografía. } \\
\text { El espacio geográfico } \\
\text { europeo y español. } \\
\text { 4.1. La Unión Europea. }\end{array}$ \\
\hline & & $\begin{array}{l}\text { 4.2. La diversidad } \\
\text { geográfica de España. } \\
\text { 4.3. El Estado espa- } \\
\text { ñol. } \\
\text { Bloque 5. Geografía. } \\
\text { El espacio geográfico } \\
\text { de la Comunidad de } \\
\text { Madrid. }\end{array}$ \\
\hline
\end{tabular}

23 La confusión puede estar dada por los objetivos del curso, pues la relación es mucha. Ver: Decreto 23/2007 del 10 de mayo de 2007, expedido por el Consejo de Gobierno, mediante el cual se establece para la Comunidad de Madrid el currículo de la Educación Secundaria Obligatoria.

24 Es necesario recordar que en el cuarto curso no hay bloque relacionados directamente con la Geografía, pues, así como en el tercer curso la Geografía ocupa varios, la Historia lo hace en el cuarto curso. 
Algo de gran valor para el alcance de tales metas, entendidas como el pleno desarrollo de los contenidos, es la manera como se expresan la evaluación, la metodología y la didáctica, al menos en la norma. Se enfatizan en el sentido de los procesos de enseñanzaaprendizaje como reforzados por diversos recursos, incluida la idoneidad de los proyectos educativos y la autonomía de los departamentos de Geografía e Historia en las instituciones para la ordenación de tales procesos. Desde lo pedagógico, esto deja una idea rica y bastante completa. Aunque esto puede ser sinónimo de indefinición para algunos tanto como apertura de horizontes para otros. Esto se explica en que, al realizar la evaluación, por lo menos se tienen en cuenta la evolución psicopedagógica y el desarrollo mental de los alumnos, así como para el desarrollo de la asignatura se tiene en cuenta la construcción progresiva de conocimiento, sin dejar de lado la importancia de la práctica en el manejo de herramientas y técnicas para el estudio de diferentes aspectos.

Lo anterior no sonaría más que a una agregación entre psicología evolutiva piagetiana, constructivismo y algo de pestalozzianismo, una visión fragmentada de la teoría de la educación, aunque desde la perspectiva contemporánea y del aprovechamiento de los recursos de diversos enfoques, desde una visión articuladora y compleja, es válido e interesante. Eso es lo que establece la norma para la Comunidad de Madrid en general; falta ver la manera como se aplica, teniendo en cuenta la posible diversidad en el tipo de proyecto o propuesta educativa que pueden tener las diferentes instituciones.

Los libros de texto son la herramienta más clara para contrastar no solo el cumplimiento de la norma, sino la visión conjunta de lo que se entiende por Geografía y ciencias sociales, dado que la norma establece que deben estar en concordancia con el currículo aprobado por la comunidad autónoma y por otros entes de control territorial25. Esto constituye un

25 Ver Disposición adicional segunda, en: Orden 3320-01/2007, de 20 de junio, del Consejero de Educación, por la que se regulan para la Comunidad de Madrid la implantación y la organización de la Educación Secundaria Obligatoria derivada de la Ley Orgánica 2/2006, de 3 de mayo, de Educación. inconveniente, dado el carácter reciente de la norma, pues el hecho de que solo haya estado vigente desde hace dos años y medio dificulta su plena aplicación en los textos. Se podría realizar una comparación si se tiene en cuenta que la normativa anterior tampoco tenía un número significativo de años de aplicación ${ }^{26}$. Esto es significativo desde diferentes perspectivas: por un lado, proyecta el interés de las autoridades en transformar y adecuar constantemente las políticas educativas a las exigencias de las sociedades actuales, mientras algunos pueden verlo como la carencia de horizontes claros para un proyecto de educación nacional. Hay que aclarar que tal opinión no podría considerarse para este caso, dada la razón primordial de que la nueva Ley Orgánica de Educación española está relacionada con su articulación al sistema de la Unión Europea.

\section{Las otras disciplinas y los textos escolares}

En España han sido publicados diversos textos sobre las ciencias sociales en la ESO, primordialmente orientados a servir de guía al profesorado en el desarrollo de su actividad. Casi en su totalidad, estos textos estaban vigentes con la normativa anterior, pero en ellos se encuentran patrones subsistentes, como la preocupación por las orientaciones, la evaluación, los recursos documentales y los ejercicios de articulación entre temas propios de las ciencias sociales, en los que convergen diversas disciplinas. Cabe mencionar que aspectos básicos como la evaluación son explicitados muy claramente en la norma, aunque no estén muy detallados.

- Geografía

- Historia

- Ciencias Sociales:

-Economía

-Antropología

-Ideas y formas políticas

-Sociología

26 La normatividad anterior radica en la Ley 14/ 1970, publicada en el BOE número 187, del 4 de agosto de 1970; y su reforma mediante la Ley Orgánica General del Sistema Educativo (LOGSE), de 3 de octubre de 1990 (publicada en el BOE de 4 de octubre), promulgada por el gobierno socialista de 1990. 
En un caso muy particular, que puede acercarnos al interrogante inicial de la Geografía escolar en relación con las ciencias sociales, se encuentran, además de orientaciones didácticas generales y especificas, una clasificación que confirma la idea de esta disciplina como un cuerpo al que colaboran algunas ciencias humanas, dentro de las cuales se incluye a la Geografía y la Historia. En este sentido, llama la atención encontrar la siguiente clasificación en una sección de guía documental y de recursos27.

Este texto de orientación al profesorado es una guía para maestros que permite apreciar la noción de ciencias sociales y el vínculo de la Geografía con este cuerpo disciplinar, sobre lo que se hace una recomendación para articularla con las ciencias sociales. Esta recomendación consiste en mantener articulada o actualizada la Geografía escolar, con los avances de la disciplina a nivel general, lo que asegura que se posibilitará el progreso en aspectos relacionados con la didáctica de esta disciplina en las aulas. Esto constituye un consejo que de ser aplicado totalmente sería peligroso, dependiendo del profesor y del curso de la ESO al que se haga referencia, pues dado el enriquecimiento teórico de la disciplina, es posible generar enfoques muy cerrados, así como confusiones conducentes a mostrar la Geografía como una disciplina carente de claridad epistémica.

Ya desde 198928 se hacían algunos cuestionamientos sobre la manera de entender las ciencias sociales en la ESO:

No puede aceptarse de ninguna manera que bajo el nombre de Ciencias Sociales se estén impartiendo en el primero de los niveles citados exclusivamente conocimientos mal integrados de Geografía, Historia e Historia del arte. A la tradicional enseñanza de estas materias debería también añadirse la de otras ciencias sociales:

27 Tomado de: Ministerio De Educación Y Ciencia. (1992). Secundaria Obligatoria: Ciencias Sociales, Geografía e Historia. Madrid. (Pp. 185).

28 Antes del las dos últimas normativas, la Ley de Ordenación General del Sistema Educativo (LOGSE) de 1990 y la Ley Orgánica de Educación (LOE) de 2006.
Sociología, Antropología, Economía y Ciencia Política ${ }^{29}$.

La opinión citada coincide con la aparición de la Ley de Ordenación General del Sistema Educativo (1990), que según algunos transformó radicalmente el sistema educativo español que tenía una normativa de los años 70. Capel, quien hace tal apreciación, se preocupa por la articulación de la Geografía al concepto o conjunto de las ciencias sociales. Esta cuestión es aún un poco oscura, por decirlo de algún modo, pues no hay claridad sobre la pertinencia del enfoque que se le da a esta articulación, ni sobre el concepto o idea de ciencias sociales. Es importante reconocer que en el momento en que el autor hace tal aseveración se imparte como ciencias sociales Geografía, Historia e Historia del arte, que es lo que en la actualidad se sigue concibiendo como ciencias sociales en los departamentos de Didáctica del mismo nombre, de las facultades de educación españolas, reconociendo en algunos casos que esa idea es un poco cerrada, humanísticamente parcelada.

Retomando el tema de los textos escolares, lamentablemente estos no conocen o conciben los bloques comunes establecidos por la normatividad ${ }^{30}$ para el desarrollo de cada curso, lo que nos muestra a las ciencias sociales algo distantes, dejando a la Geografía y a la Historia como un matrimonio de tipo formal. La manera como se plantean las unidades con sus temáticas deja ver una preocupación por ajustarse a lo que dice la norma, no por cumplirla como tal. Se evidencia un trasfondo que muestra la necesidad de reinar y vender en el mercado, por lo que la cuestión disciplinar siempre genera inquietudes y sinsabores para quienes lo vemos desde la educación superior, sea cual sea nuestra misión.

Se manifiesta una cierta concordancia entre la norma y los textos en el primer y segundo curso,

29 Capel, H. y Arteaga, L. (1989). La Geografía en un curriculum de ciencias sociales. En M. Carretero et al, La enseñanza de las ciencias sociales (pp. 75-102). Madrid: Ediciones Visor.

30 Para este caso, me refiero al Decreto 23/2007 del 10 de mayo de 2007, expedido por el Consejo de Gobierno, mediante el cual se establece para la Comunidad de Madrid el currículo de la Educación Secundaria Obligatoria (publicada en el BOE, 30 de noviembre de 2007) 
pero en el tercero y cuarto esta tendencia parece cambiar. Esto se evidencia en que especialmente el texto para el tercer curso, en el que priman los contenidos de Geografía, ha sido nombrado por algunas editoriales como Geografía 3, o se ha especificado, de alguna manera, en el título el predominio de esta disciplina. Un elemento para reflexionar es que para el cuarto curso, cuyos contenidos primordialmente son de historia, no se habla de esta disciplina sino de Ciencias Sociales 4.

Si nos preguntamos sobre el potencial interdisciplinario de la Historia con relación al resto de las ciencias sociales, puede decirse que esta acoge un poco a la Antropología, que se ligaría algo con la Política y la Sociología. Cuando se habla, por ejemplo, de la llegada de la democracia a España, lo que debe estar claro es que las ciencias sociales son más que la parcela de estos semivínculos, casi inexistentes, lo que resulta grave para el futuro de este amplio cuerpo disciplinar. En la ESO está la posible solución: hay que mostrar las ciencias sociales tal y como son, no fragmentadas, sino bastante claras. Por lo menos se puede iniciar dando un primer paso hacia la concreción de la manera como se concebirá el área en los cursos de la ESO, los bloques y su articulación o interdisciplinariedad.

Podríamos decir que tanto Geografía como Historia son interdisciplinares, que la historia se relaciona con la Historia del arte y otras disciplinas, así como la Geografía lo hace de modo natural con la demografía y la Economía, e incluso con algo de las ciencias naturales. Pero vemos que Geografía e Historia permean muchos campos, pero no en todos de igual manera. Ahí está la cuestión por la cual resultaría fácil decir que eso es interdisciplinariedad, sin conectar a los jóvenes con la más mínima aproximación a la esencia de esas otras disciplinas, negándoles la posibilidad de que construyan una panorámica completa de lo que son las ciencias sociales, e incluso negándoles la posibilidad futura de que se conviertan en artífices de la generación de conocimientos nuevos. Estaríamos negando así la pertinencia de otras disciplinas en la educación superior, algo que parece no ser tan grave como mantener minimizada la posibilidad de una sólida noción de mundo y de humanidad en los jóvenes.

En general, los textos escolares están ajustados a la normativa, pero aunque tratan de seguir manteniendo la idea de esta, no la proyectan de manera idéntica. Este aspecto también podría ser explicado por el interés de las editoriales en mantenerse vigentes ante las demandas del profesorado y de los institutos de educación secundaria. Puede valorarse la manera como en los textos de algunos grupos editoriales se plantea, al menos en la parte de contenido o desglose de unidades y temas, la posible relación de estos con competencias básicas relacionadas con educación ambiental, educación para la paz, moral y cívica, los derechos humanos y la igualdad de género. Por desgracia para la Geografía, aunque sea vista desde una manera integrada con la economía o la sociología, solo se le establece como posible su conexión o asociación con la educación ambiental. Cabe mencionar que para el resto de competencias o ejes, es la historia y los contenidos que de ella se derivan los que poseen la potestad de ser considerados articuladores o transversales, por no decir interdisciplinares. En los textos, además, se aprecia que la suma de contenidos propiamente económicos y demográficos debe entenderse como geográficos.

Podríamos asegurar que un verdadero aire de ciencias sociales se evidencia en secciones como las cartillas para el tercer curso, complementarias a los libros de texto que anexan algunas editoriales. En ellas que se trata de proyectar el contexto inmediato desde una totalidad que, por lo general, se estructura en dos bloques: uno enfocado hacia la geografía humana de Madrid, en donde se proporciona una mirada a la comunidad desde las ciencias sociales, teniendo en cuenta la población, las ciudades, la organización social y política y sus áreas metropolitanas. Todo esto se hace desde la claridad que posibilita la unificación de contenidos, para acabar con una revisión a los territorios de la Comunidad de Madrid en diversos momentos de la historia que figuran en el cuarto curso, el cual en su mayoría está compuesto solo por esta disciplina. 
Desafortunadamente, siempre han existido jerarquías dentro de la amplitud de esta área. Prueba de ello es la tendencia hacia las articulaciones con otras disciplinas e incluso la prevalencia de otras denominaciones sobre las mismas, además del papel de la Geografía y la Historia, aunque algunos las consideren ciencias humanas. Por ejemplo, para muchos tiene más sentido una historia económica o una geoeconomía, que una socioantropología o una sociología. Los patrones de parcelación, dar primacía de una disciplina sobre otra son sustentados por algunos en la inexactitud de las mismas, en la poca cientificidad, en las dificultades para explicar su método y objeto de estudio, y en fin, en miedos que lo único que dejan claro son vacíos; miedos a lo desconocido. Allí están los juicios a priori que la humanidad aún no ha podido superar. Desgraciadamente, si no los supera la academia, el profesorado, las universidades, ¿ cómo llegará tal superación a las escuelas, colegios e institutos? Veamos una apreciación sobre este aspecto:

El área de Ciencias Sociales, Geografía e Historia, tal como aparece en el diseño curricular base para la educación secundaria obligatoria está constituida por las siguientes disciplinas: Historia, Geografía, Historia del arte, Ciencias Políticas, Economía, Sociología, Antropología y Psicología.

En realidad... puede observarse un claro predominio de la Geografía y la Historia. En un segundo plano aparecen la Historia del arte, la Economía y la Antropología. Y en un tercer plano las Ciencias Políticas, la Sociología y la Psicología. Esta ultima prácticamente no tiene presencia explicita ${ }^{31}$.

De modo bastante evidente se aprecia esa jerarquía de unas sobre otras. Es bastante evidente esta jerarquía, que atenta contra la esencia de las ciencias sociales y es más notoria en el momento en el que analizamos un libro de texto o revisamos los contenidos, sea en la norma o en planes de clase. Este hecho parece no ser una preocupación para

31 Sánchez, P. (1992). Sobre la enseñanza del área de Ciencias Sociales. En A. Monclus, La enseñanza de la Historia, la Geografía y las Ciencias Sociales (pp. 43-76). Madrid. Editorial Complutense. todo el profesorado, ni para muchos de los docentes universitarios. Al examinar un poco más el texto citado podemos pensar sobre la estructura, o al menos sobre lo que aparece como base: está clara la potestad de la Historia que, con la Historia del arte, consigue ocupar un espacio significativo, con lo cual se liga a una gran parte de la Antropología, así como la Geografía lo hace con la Economía y con una parte de la Sociología, ya que lo concerniente a las ciencias políticas suele ser entendido como historia.

Así, quedaría por estudiar si ciertamente estas disciplinas tienen presencia en las aulas, por mínima que sea, pues una cosa es ligar o articular, y otra muy diferente es hacer referencia o citar. Ante tal diferencia surge una inquietud: ¿Cómo hallar hilos conductores que permitan la visibilización de cada una y de modo no aislado al concepto integrador de las ciencias sociales? Ese es el reto. Pero valdría la pena cuestionarse sobre esa psicología a la que hace mención el autor. ¿Será la psicología la que debe contribuir a la construcción del ciudadano? ¿Será la psicología para ver y entender las ciencias sociales mismas como un todo? ¿Tendrá esto algo que ver con la educación para la ciudadanía? Las respuestas a esto no pueden buscarse solo en los académicos e investigadores. La respuesta está en las escuelas, en los profesores y sus estudiantes, en lo socialmente construido como ciencias sociales, en el imaginario cercano y vital: la praxis.

Algo muy particular es el tema de la educación para la ciudadanía, denominada por la normativa como "Educación para la ciudadanía y los derechos humanos". Tal cuestión es la relación existente entre la misma y la "Educación Ético-Cívica", vistas en el segundo y cuarto curso de la ESO, respectivamente. ¿No hay relación? Si esta última es impartida por un profesorado con formación en filosofía, ¿no debería ser filosofía como tal? ¿Podría entenderse fácilmente que la primera va centrada hacia los derechos humanos y la segunda hacia una formación filosófica de la vida que contribuya a la formación de los jóvenes? Analicemos algunas apreciaciones sobre la educación ciudadana:

La educación del ciudadano se entiende como desarrollo de la capacidad de percepción social 
como base para la comprensión racional de los fenómenos de relación entre los hombre de una determinada comunidad (Cfr. Shantz, 1990)32.

En buena medida, la educación del ciudadano se suele convertir en una transmisión o repetición de tópicos e ideas susceptibles de tener o no tener significación en el tiempo en el que se enseñan ${ }^{33}$.

Las aseveraciones anteriores dan una clara idea de la complejidad de las ciencias sociales y de todo lo que tiene que ver con ellas, la amplitud de horizontes a los que tienen que responder por obligación ética y científica y las dificultades a nivel académico-disciplinar que esto conlleva. He aquí un inconveniente más que los profesores deben sortear en las aulas día a día, el carácter social como tal, que radica en el manejo particular de las asignaturas, dado por lo actores del proceso educativo. Si se sigue profundizando en esto, encontraremos mayor número de dudas por resolver y batallas por librar que, si son tomadas con racionalidad positivista, alteran el equilibrio armónico y humanístico de lo social. Podemos encontrar un ejemplo claro de esto al revisar un libro sobre la enseñanza (Grupo Ínsula Barataria, 1994) de las ciencias sociales: la educación para la familia como algo clave para esta enseñanza. Esto se puede considerar realmente bueno en la medida en que se articula con la Antropología, debido a que se hace mención a su estructura antropológica. Esto es válido, como muchas otras cosas, pero la validez, así como la humanidad en general, seguirán siendo nociones relativas.

\section{De los recursos}

Luego de la visita a algunas instituciones educativas, en las conversaciones con estudiantes y docentes por separado, se logró evidenciar el marco de la necesidad que tienen las editoriales de imponerse. Se pueden resaltar estrategias significativas como la recurrencia al uso de material computarizado, tanto para el enriquecimiento de la labor docente, como

32 Citado en: García Hoz, V. (1996). Enseñanza de las Ciencias Sociales en educación secundaria. Madrid: Ediciones Rialp.

33 Ibid. pp. 29. para la profundización en temas y el desarrollo de otras actividades por parte de los estudiantes. Así mismo, el común uso de material cartográfico al final de los textos para apoyar el desarrollo de los procesos en las aulas, ayuda a la articulación de estas dos disciplinas, aunque no deja clara tal colaboración. Esta colaboración puede y debe ser responsabilidad del docente, pero solo si este entiende los textos como un recurso más.

A mi parecer, algo muy particular en la manera como se estructuran los textos, que evidencia una leve pasividad de los maestros, es el modo como se clasifican los procesos a partir de conceptualizaciones constantes como relación, síntesis, aplicación, ampliación, e incluso los parámetros en los que está planteado el texto del profesor, el cual resuelve todas las actividades de una manera que no es la ideal, convirtiéndolo que convierte el quehacer educativo en algo operativo y de poca significación. Sin embargo, hay que mencionar la dilemática dependencia del profesorado, dada la idoneidad de las actividades y talleres planteados en algunos textos que vienen acompañados de crónicas, reseñas y demás herramientas para manifestar un desarrollo activo de la asignatura. Pero lo realmente importante es contrastar el uso del libro según los actores del proceso, estudiantes y maestros, tarea que se desarrollará más adelante.

Algunos elementos peculiares en los textos que -creo- invitan a cualquier maestro, sea o no de ciencias sociales, a pensar lo social, a reconocer la pertinencia de las mismas, a legitimar esos saberes que son inherentes al concepto de humanidad que definimos día a día, desde el mundo en general y desde las aulas en particular, son aspectos como la presencia de los cuadernos de atención a la diversidad, y decimos: ¿dónde si no en las ciencias sociales?

Para abordar estas cuestiones, en estos cuadernillos que acompañan a los textos se habla de Madrid, más que histórica y geográficamente, como un escenario de construcción ciudadana y como un espacio social que quiere vincular a esos jóvenes. Así, estos cuadernillos ofrecen al maestro una ayuda educativa de gran valor, sea para refuerzo o profundización, $\mathrm{y}$ un significativo potencial intercultural que hace 
de la constante inmigración un problema menos grave para la educación española. Esto le brinda a las ciencias sociales la oportunidad de trabajar la inclusión para dar ese sentido a las competencias básicas del currículo, entre las cuales es resaltada la "social y ciudadana 34 ".

\section{Sobre la enseñabilidad de la disciplina}

Entendiendo la didáctica en el sentido del método y los recursos para la enseñanza, vale la pena mirar detenidamente el caso de la Geografía escolar en España, reconociendo que en líneas anteriores ya se había mencionado la especificidad conceptual un poco condicionante de los procesos de enseñanzaaprendizaje de esta disciplina. Esta especificidad es evidente en los textos y ratificada en la conservación de principios base como el desarrollo mental y la evolución psicopedagógica, lo que recuerda la tradición en la enseñanza de esta disciplina y la muestra como algo que requiere técnica y análisis asociados a las prácticas de campo, al uso de mapas y a la abstracción lógica.

En la actualidad, esa lógica es la que permea las concepciones sobre la enseñabilidad de esta disciplina, desde la manera en la que podemos entender la programación, el uso de medios interactivos y los talleres cartográficos. Desde los textos, estos recursos se ven como algo de uso común para el análisis de aspectos tanto geográficos como históricos, así como los llamados bancos de actividades, los contenidos en los textos guía y los textos de los docentes. Todo esto nos lleva a pensar en posibilitar desde el aula la relación y síntesis conducentes a la aplicación, en una etapa en la que los jóvenes deben estar en capacidad de reconstruir conocimiento, y dejando la puerta abierta para la ampliación y el descubrimiento de elementos aún más transversales a los recreados en el aula. Esa es la aventura que se emprende al enseñar; ese es el ideal que muestran algunos textos en su estructura.

34 Competencia social y ciudadana. Ver: Real Decreto 1631/2006 del 29 de diciembre, por el que se establecen las enseñanzas mínimas correspondientes a la Educación Secundaria Obligatoria (publicado en el BOE, 5 de enero de 2007).
Hasta hace un tiempo, tiempo continuo y aparentemente infinito para muchos de nuestros jóvenes, la palabra Geografía hacía referencia a nombres de sitios, lugares, hechos y estructuras. Según las corrientes pedagógicas de renovación, desde hace aproximadamente 20 años e incluso más, hay otra Geografía, apoyada en la relación y/o articulación, la extensión y la causalidad de los hechos geográficos. Estos tres grandes aspectos, que se pueden entender como lemas, son la base para el progreso hacia una noción de ciencias sociales que vincule más la Geografía; en otras palabras, sería una Geografía que permee más todo lo que percibimos como ciencias sociales. Para tener una mejor aproximación hace falta dar un vistazo a la práctica, a la realidad.

\section{En la práctica}

El contacto con la realidad educativa se llevó a cabo en diversas instituciones de la fundación FU$\mathrm{HEM}^{35}$, la cual responde por la administración de escuelas y/o colegios de tipo concertado, es decir semipúblicos, en los que predominan estudiantes de diversas características sociales y académicas. Esto posibilitó una aproximación a la situación actual de las ciencias sociales y de la Geografía en particular, en las aulas de Madrid, desde el uso de entrevistas informales hasta charlas con profesores, estudiantes y jefes de departamento.

El uso de los textos es muy variable para los docentes, según los intereses de cada uno y las metas que pretendan alcanzar con el curso. Cabe recordar que la selección de textos de una editorial u otra se realiza de manera discutida según las necesidades temáticas establecidas por los docentes del área, quienes se agrupan en departamentos en el interior de cada colegio o instituto. Así mismo, los docentes se refieren a la interdisciplinariedad y al concepto de ciencias sociales. Desde diversas perspectivas, ellos también conciben la norma como algo que hay que cumplir, aunque no esté claro; un ejemplo inme-

35 Fundación FUHEM es una institución privada e independiente, sin ánimo de lucro, que trabaja por la construcción de una sociedad más justa y solidaria, a través de la acción educativa y la promoción del debate y el análisis de cuestiones ecosociales. Mayor información en: http://www.fuhem.es 
diato es el trabajo por competencias, el cual, según muchos profesores, nadie logra tener claro. Algunas instituciones intentan aproximarse a la construcción de un modo de aplicar este concepto a la enseñanza en la ESO; algunos institutos alcanzan a trazar metas y desarrollarlas a lo largo de año, articulando estos conceptos con el proyecto educativo institucional, de manera que se plantean trabajos comunes entre departamentos para el logro de avances conjuntos.

$\mathrm{Al}$ cuestionar a algunos profesores de ciencias sociales sobre el campo especifico de la Geografía y la interdisciplinariedad en su asignatura, aseguran que el problema radica en las directrices y que si la norma, a pesar de su flexibilidad, no fracturara tanto, los contenidos de "Ciencias Sociales: Geografía e Historia” de manera disciplinar, se podría construir un panorama claro, más crítico y más reflexivo en los estudiantes de la ESO. En este panorama la significación sería algo latente, producto de la plena relación establecida por los alumnos entre los contenidos de un curso y otro.

Sobre la Geografía en particular, explican su proyección como una disciplina que se puede articular claramente con otras, dada la inclusión de temas demográficos y económicos al interior de sus bloques temáticos, lo que no se evidencia tan claramente para el caso de la Historia. Sin embargo, aquí la influencia del profesor es decisiva: si desea mostrar al estudiante las ciencias sociales como un escenario amplio, tiene la potestad de hacer referencia a otras disciplinas que hacen parte de las ciencias sociales o seguir ayudando a mantener la idea de que tal constructo es igual al compendio de Geografía, Historia e Historia del arte. Lamentablemente, esta última idea es la que se mantiene en gran parte de la educación superior. Muchos profesores aseguran que si a la asignatura antes mencionada se le quitase el término de ciencias sociales y quedara solo como Geografía e Historia, sería igual para lo que se desarrolla y cómo se desarrolla en las clases, todo a causa de la norma y la presión de contenidos que desde aquella se ejerce.

La enseñanza de la Geografía en particular, en el marco de las ciencias sociales, no parece tener grandes problemas para los docentes, quizás por la manera sesgada como están establecidos los contenidos ${ }^{36}$ y la poco evidente necesidad de articulación con otras disciplinas. En ellos se hace mención al valor potencial que se le agrega a los cursos donde la asignatura es impartida por licenciados en Geografía, quienes en su mayoría hacen un buen trabajo con esta disciplina, al construir en las aulas sus propios materiales de trabajo, al intentar tratar a temas de trascendencia y relevancia y al buscar la formación critica en el estudiantado, aunque haya que evadir un poco la norma. Aquí se puede mencionar el interés de algunos profesores en la formación sobre el mundo, no limitada al entorno ${ }^{37}$.

Como aspecto relevante, podríamos decir que las salidas pedagógicas son una herramienta clave para la formación, son planeadas por cada departamento en el interior de los colegios e institutos y se realizan sin ningún problema, algunas veces con el apoyo de otro profesor, de manera regular y en trasporte público. Además, están las salidas de fin de curso, normalmente se realizan para cada nivel en la mayoría de las instituciones, dado que estas también son aprovechadas ocasionalmente por el profesor de "Ciencias Sociales: Geografía e Historia" para afianzar las temáticas desarrolladas en clase.

Solo pocos profesores son conscientes de las dificultades de base, como la articulación de la Geografía física con la vida diaria. Ese es uno de los retos de la Geografía desde hace mucho tiempo, ante el cual muchos aseguran que los conceptos básicos de temas como el ordenamiento no sirven para que los estudiantes entiendan la pertinencia de lo físico. A esto se puede agregar la coincidencia de contenidos con las ciencias naturales al menos en el primer curso de la ESO. Ante esto, muchos profesores aseguran trabajar en conjunto con su colega de ciencias naturales, para no dictar el mismo tema,

36 Me refiero a la estructura por bloques, unos de Geografía y otros de Historia, además de la forma como se plasma la continuidad de unos y otros a lo largo de los cuatro cursos de la ESO.

37 Esto se explica en la resistencia de algunos profesores a dar prioridad a los contenidos relacionados con Madrid, España y la Unión Europea sobre contenidos de mundo en general, dado que este enfoque busca sobre todo priorizar en el conocimiento de la comunidad, en este caso Madrid, en concordancia con la Unión Europea. 
mientras otros aseguran recurrir a la socialización con los muchachos a partir de lo que estos ya dicen haber visto, aprovechándolo para la construcción de un enfoque eminentemente geográfico.

Pero, si hay algo que puede dar claridad en lo que consideramos como el mayor problema de la enseñanza de las ciencias sociales en la ESO, no solo en Madrid sino en toda España, es la formación del profesorado ${ }^{38}$ para esta área, dado que no existe una formación específica a nivel universitario. Recordemos que el nombre completo de esta asignatura es: "Ciencias Sociales, Geografía e Historia" y que recientemente se extinguió el programa de formación universitaria de "Licenciatura en Geografía e Historia", que marcaba la pauta para que la academia se pensara las ciencias sociales. Si eso era ciencias sociales, ¿qué son hoy, cuando casi todo profesional español con una licenciatura aproximada a estas disciplinas puede ser profesor de esta área, desde Sociología, Antropología y otras? Además de la bifurcación de este programa, que fue reemplazado por los de "Licenciatura en Historia" y "Licenciatura en Geografía", ¿cómo hace el profesorado para construir su visión de ciencias sociales? Está claro que de una $u$ otra forma prima el enfoque particular, lo que explica cómo desde cada profesión se puede sintetizar de distintas maneras una idea de Geografía. Muestra de ello es que muy pocos profesores elaboran material didáctico en el aula con los estudiantes; quienes lo hacen son en general quienes tienen formación inicial en esta disciplina. Sin embargo, la manera como participa la Geografía puede dar una visión particularmente crítica para entender la realidad global.

Esta cuestión sobre la formación del profesorado es vital. Su solución no radica sólo en los ejercicios de investigación en las universidades y en las aulas; es necesaria la participación activa de diversos sectores para que profesores con formación en Historia, Geografía y cualquier otra disciplina, se integren

38 Ver, sobre la formación del profesorado: Diez Ros, R. (2000). La formación docente en la licenciatura de Geografía. En J. Gonzáles y M. Marron Gaite, Geografía, Profesorado y Sociedad: Teoría y Práctica de la Geografía en la enseñanza (pp. 57-62). Murcia: Compbell. a la comprensión de las ciencias sociales como un constructo amplio que no se a reduce la Historia y la Geografía. Solo así habrá un acercamiento a la idea de ciencias sociales, por lo menos en España, dado que en otros países tanto Historia como Geografía simplemente van incluidas en tal noción, así sean disciplinas claves.

Por un lado, están los académicos que aseguran que la Geografía y la Historia son ciencias humanas, no sociales. Por otro, están quienes dicen que Geografía e Historia son ciencias humanas, pero aseguran que nada mejor que ellas para acercarnos a una idea de ciencias sociales. Por último, existe un número reducido de profesores y uno amplio de alumnos que asegura que las ciencias sociales son la Geografía y la Historia.

Estaría bien aclarar que en la formación de maestros (entiéndase la docencia para educación primaria en algunas facultades de educación) se llega a ser consciente de que en las ciencias sociales hay otras disciplinas como la Sociología y la Antropología, principalmente. Aun así, para el estudiantado de la ESO, ciencias sociales es igual a Historia y Geografía, lo que evidencia que no hay claridad en la idea o noción de ciencias sociales en España, y esto es proyectado en el sistema educativo. Da la impresión de que ni los grandes académicos se preocupan por estas cuestiones, aparentemente insignificantes, pero cuyas ambigüedades tienen mucho impacto. Si esto fuese comparado con países como Colombia, donde la Geografía ocupa un papel menor en los contenidos de ciencias sociales en la educación secundaria, los geógrafos y amantes de esta disciplina se sentirían muy satisfechos con la reivindicación en la escuela del carácter social de su disciplina, mientras quienes comprenden las ciencias sociales como un conjunto para interpretar el mundo y la presencia en él, manifestarían su desacuerdo.

\section{Conclusiones}

No es nada sencillo intentar concluir, pues se trata de una aproximación a lo que podríamos llamar el imaginario de las ciencias sociales, teniendo como referente particular la Geografía. La tarea está hecha solo si estas páginas han servido para 
generar cuestionamientos e inspirar a investigaciones completas y profundas, que aclaren ese borroso y delicado tema de lo disciplinar en las ciencias sociales, aun para quienes pretendan cuestionarse más sobre la Geografía en la escuela. Sin embargo, se hacen necesarias algunas apreciaciones que podrían clarificar un poco el panorama:

- La normativa educativa española contempla la ESO como una etapa de gran importancia para la formación de los jóvenes, por lo que las ciencias sociales, vistas como la asignatura "Ciencias Sociales Geografía e Historia", mantienen un estatus similar a cualquier otra área del saber, con contenidos específicamente ligados a estas dos disciplinas, también enmarcadas en las competencias básicas establecidas por la Unión Europea.

- El carácter reciente de la implementación de la nueva normatividad educativa (Ley Orgánica de Educación -LOE- de 2006) no ha permitido que el profesorado de la ESO llegue a consensos sobre su comprensión y adopción. Sin embargo algunos colegios y/o institutos intentan desarrollar de forma paulatina algunos avances en la aplicación de la misma, además de la autonomía de las instituciones para ajustar la normatividad a su proyectos institucionales.

- La Geografía, al igual que la Historia, ocupa un espacio importante en el currículo y, por tanto, en el concepto de ciencias sociales en la ESO. Esto da la posibilidad al profesor de hacer alusión a la existencia de otras disciplinas en el marco de las mismas, aspecto de difícil alcance pues no todo el profesorado tiene una visión de esta área más allá de la Historia y la Geografía. Dicha limitación obedece a su formación, generalmente en una de estas disciplinas, e influye directamente en la manera como desarrollan sus clases y el uso de herramientas didácticas.

- El papel de la Geografía como disciplina clave posibilita su desarrollo como disciplina social, al incluir temáticas relacionadas con la demografía y la economía, particularmente en el tercer curso de la ESO. Persiste la dificultad para resaltar la vinculación de los contenidos de Geografía física, como algo social. Adicionalmente, el proceso de enseñanza de contenidos geográficos a lo largo de la ESO es muy fragmentado, lo que dificulta una construcción conjunta de la disciplina o de las ciencias sociales en los jóvenes.

- En algunos casos los materiales computarizados de los libros de texto son usados según las preferencias del profesor, aunque son seleccionados, así como los contenidos y las salidas a desarrollar, por los departamentos. Estos están constituidos por las distintas áreas del saber en cada institución y son fuente clave para la ejecución del curso. Así, queda en potestad del docente el direccionamiento total de la idea de ciencias sociales.

- Para el profesorado y muchos académicos universitarios, el concepto de ciencias sociales comprende Geografía, Historia e Historia del arte, lo cual es un legado de la anterior Ley General de Educación ${ }^{39}$, que aún persiste en las universidades. Adicionalmente, fuera de estos espacios, en los centros de investigación por ejemplo, la Geografía y la Historia son ciencias humanas y las ciencias sociales son otras. Los profesores responden de manera diversa a esta apreciación.

- Es evidente la necesidad de revisar la disciplinariedad en las ciencias sociales, para construir una visión conjunta entre científicos, académicos y escolares. Solo así se alcanzará una significación apropiada para este cuerpo disciplinar y se logrará redireccionar procesos articulados a las construcciones sociales del conocimiento que propicien el rescate de lo humano, sin fragmentaciones ni perspectivas particularizadas.

39 Se hace referencia a lo establecido por la ordenación general de sistema educativo, mediante la -LOGSE-LEY ORGÁNICA 3-10-1990, NÚM. 1/1990 JEFATURA DEL ESTADO, BOE 4-10-1990, NÚM. 238, [PÁG. 28927] 


\section{Referencias}

\section{Normativa}

Ley General de Educación de 1970: Ley 14/1970, de 4 de agosto, General de Educación y Financiamiento de la Reforma Educativa. Publicada en el BOE núm. 187.

Ley Orgánica de Ordenación General del Sistema Educativo-LOGSE-: Ley Orgánica 3-10-1990. Publicada en el BOE núm. 238.

Ley Orgánica de Educación: Ley Orgánica 2/2006, de 3 de mayo, de Educación. Publicada en el BOE núm. 106.

REAL DECRETO 1631/2006, de 29 de diciembre, por el que se establecen las enseñanzas mínimas correspondientes a la Educación Secundaria Obligatoria. Publicado en el BOE núm. 5.

DECRETO 23/2007, de 10 de mayo, del Consejo de Gobierno, por el que se establece para la Comunidad de Madrid el currículo de la Educación Secundaria Obligatoria. Publicado en el B.O.C.M. Núm. 126.

ORDEN 3320-01/2007, de 20 de junio, del Consejero de Educación, por la que se regulan para la Comunidad de Madrid la implantación y la organización de la Educación Secundaria Obligatoria derivada de la Ley Orgánica 2/2006, de 3 de mayo, de Educación. Publicado en el B.O.C.M. Núm. 185.

\section{Textos Escolares}

Agullo, E. (2007). Ciencias sociales, Geografía 3. Madrid: Ediciones SM.

Diaz, J. (2008a). Ciencias sociales, Geografía e historia 2. Comunidad de Madrid: Edelvives.

Diaz, J. (2008b). Ciencias sociales, Geografía e historia 4. Comunidad de Madrid: Edelvives.

González, I. (2007). Ciencias sociales, 1 ESO: Geografía e historia. Barcelona: Editorial Guadiel.

Ortega, M. et al. (2007). Ciencias sociales: Geografía e historia 1. Comunidad de Madrid. Madrid: Ediciones SM.

Ortiz, J. (2009). Geografía 3. Ciencias Sociales: cuadernos eso, para trabajar ciencias básicas. Grupo Editorial Bruño.

\section{Libros y artículos}

Aisenberg, B. y Alderoqui, S. (Comps.). (1997). Didáctica de las ciencias sociales: Aportes y reflexiones. Barcelona: Ediciones Paidos Ibérica.
Andina, M. y Santamaría, G. (1986). Aprendizaje de las Ciencias Sociales. Buenos Aires: Editorial El Ateneo.

Asociación Universitaria Del Profesorado De Didáctica De Las Ciencias Sociales. (1997). La formación del profesorado y la didáctica de las ciencias sociales. Madrid: Diada Editora.

Aviles De Torres, D. et al. (1999). Unidades didácticas interdisciplinares. Madrid: Editorial La Muralla.

Bauer, L. (1976). La enseñanza de la Geografía. En EDUCACIÓN: Colección semestral de aportaciones alemanas recientes en las ciencias pedagógicas $\left(\mathrm{N}^{\circ}\right.$ 14), 95-101.

Bailey, P. (1981). Didáctica de la Geografía. Madrid: Editorial Cincel.

Benejam, P. y Pagés, J. (Coord.). (1998). Enseñar y aprender ciencias sociales, Geografía e historia en la educación secundaria. Barcelona: ICE- Universitat Barcelona- Editorial Horsori.

Benejam, P. et al. (2002). Las Ciencias Sociales: concepciones y procedimientos. Barcelona: Editorial Grao.

Capel, H. y Arteaga, L. (1989). La Geografía en un curriculum de ciencias sociales. En M. Carretero et al, La enseñanza de las ciencias sociales (pp. 75-102). Madrid: Ediciones Visor.

Cunill Grau, P. (1998). La Geografía y su enseñanza en el ámbito de los tiempos actuales. En Geoenseñanza, Vol. Especial, 13-28.

Delgado, O. (1986). Permanencia del determinismo geográfico en la enseñanza de la Geografía en Colombia. En Revista Colombiana de Educación ( $\left.\mathrm{N}^{\circ} 18\right), 98-112$.

Federación Icaria. (1997). Con-Ciencia Social: Anuario de didáctica de la Geografía, la Historia y las Ciencias Sociales. Ediciones AKAL.

García, A. et al. (1997). Didáctica de las Ciencias Sociales, Geografía e Historia en la enseñanza secundaria. Madrid: Grupo Editorial Universitario.

García, V. (1996). Enseñanza de las ciencias sociales en educación secundaria. Madrid: Ediciones Rialp.

Gonzales, J. y Marron, M. (2000). Geografía, Profesorado y Sociedad: Teoría y Práctica de la Geografía en la enseñanza. Murcia: Compobell.

Gómez, J. (2002). La Construcción del Conocimiento Social en la Escuela. Bogotá: Fondo de Publicaciones Universidad Distrital Francisco José de Caldas.

Graves, N. (1985). La Enseñanza de la Geografía. Madrid: Ediciones Aprendizaje Visor. 
Grupo De Didáctica De La Geografía (AGE). (1996a). III Jornadas de Didáctica de la Geografía. Madrid: Universidad Complutense.

Graves, N. (1996b). El Reto de la Geografía ante la reforma educativa. Madrid: Universidad Complutense.

Grupo Didespai. (1985). Por una renovación de la Didáctica de la Geografía. Barcelona: Publicacions i Edicions de la Universitat de Barcelona, Institut de Ciences de l'Educacio.

Grupo Ínsula Barataria. (1994). Enseñar y Aprender Ciencias Sociales: Algunas propuestas de modelos didácticos. Madrid: Ediciones Mare Nostrum.

Guijarro, A. y Luis Gómez, A. (1992). La Enseñanza de la Geografía: Guía Introductoria. Santander: Universidad de Cantabria.

IBER. (1996). Didáctica de las Ciencias Sociales, Geografía e Historia. Métodos y Técnicas de la Didáctica de la Geografía (No 9). Año III.

Instituto De Ciencias De La Educación. (2005). Didáctica de Geografía e Historia: Formación de Profesores de Educación Secundaria. Madrid: Universidad Complutense.

Jarolimek, J. (1980). Las Ciencias Sociales en la Educación Elemental. México: Editorial Pax.

Kolevzon, E. y Malff, R. (1968). Cómo hacer interesante una clase de Geografía. México: Unión tipográfica editorial hispanoamericana.

Lopez, A. y Valls, R. (2002). Dimensión europea e intercultural en la enseñanza de las ciencias sociales. Madrid: Editorial Síntesis.

Luis Gómez, A. (1985). La Geografía en el Bachillerato Español (1836-1970). Barcelona: Universitat de Barcelona.

Luis Gómez, A. y Romero, J. (2007). Escuela para todos, Conocimiento Académico y Geografía Escolar en España (1830-1953). Santander: Servicio de Publicaciones Universidad de Cantabria.

Marron, M. et al. (2003). La Enseñanza de la Geografía ante las Nuevas Demandas Sociales. Toledo: Universidad de Castilla- La Mancha.

Marron, M. et al. (2001). La Formación Geográfica de los ciudadanos en el cambio de milenio. Madrid: Universidad Complutense.

Martin, P. et al. (1993). Ejemplificación de unidades didácticas para el área de Ciencias Sociales, Geografía e Historia. Madrid: Editorial Síntesis.
Ministerio De Educación Y Ciencia. (1992). Secundaria Obligatoria: Ciencias Sociales, Geografía e Historia. Madrid.

Monclus, A. (1992). La enseñanza de la historia, la Geografía y las ciencias sociales. Madrid: Editorial Complutense.

Plans, P. (1967). Orientaciones sobre Didáctica de la Geografía. Madrid: Editorial Magisterio Español.

Perez, M. et al. (2002). Interdisciplinariedad, discursos sociales y enseñanza media. Cuadernos de la Facultad de Humanidades y Ciencias Sociales ( $\left.\mathrm{N}^{\circ} 015\right)$, 323340. San Salvador de Jujuy, Argentina: Universidad de Jujuy.

Rodríguez, F. (2002). Concebir la Geografía escolar desde una nueva perspectiva: una disciplina al servicio de la cultura escolar. Boletín de la A.G.E (N³3), 173-186. Madrid: A.G.E.

Santiago, J. (1997). La preservación del medio ambiente: el hombre y la enseñanza de la Geografía. En Geoenseñanza, $23-40$.

Servicio De Publicaciones Del Ministerio De Educacion. (1981). Ciencias Sociales. Estudios y experiencias educativas. Madrid: Ministerio de Educación.

Souto, X. (1998). Didáctica de la Geografía: Problemas sociales y conocimiento del medio. Madrid: Ediciones del Serbal.

Ubieto, A. et al. (1999). Aspectos didácticos de ciencias sociales. Zaragoza: ICE. Universidad de Zaragoza.

UNESCO. (1966). Método para la enseñanza de la Geografía. Barcelona: Editorial Teide.

Zurbano, J. (1997). Diseño de las áreas de enseñanza en educación secundaria obligatoria. Madrid: Editorial Escuela Española. 